\title{
Análisis comparativo de los precios de los medicamentos en América Latina ${ }^{1}$
}

\author{
Roberto Álvarez y Aldo González
}

\section{Resumen}

En este trabajo se comparan los precios de medicamentos en las seis mayores economías de América Latina. Utilizando un modelo econométrico de panel con efecto fijo por país y controlando las variables referentes a las características de los fármacos, la comparación abarca 19.741 unidades comercializadas durante el período 2010-2015 y se efectúa a nivel mayorista y minorista y según el tipo de medicamento (innovador, similar y genérico). A nivel agregado y en salida de farmacia, la clasificación de más barato a más caro es: $1^{\circ}$ Perú, $2^{\circ}$ México, $3^{\circ}$ Argentina, $4^{\circ}$ Chile, $5^{\circ}$ Colombia y $6^{\circ}$ Brasil. En medicamentos innovadores, la Argentina y el Perú presentan los menores precios. En similares, México y la Argentina tienen los precios más bajos, mientras que para genéricos puros, el Perú y Chile serían los más baratos. La clasificación no cambia sustancialmente si se comparan los precios a la salida de laboratorio.

\section{Palabras clave}

Industria farmacéutica, productos farmacéuticos, precios, análisis comparativo, modelos econométricos, datos estadísticos, América Latina

\section{Clasificación JEL}

L16, L65

\section{Autores}

Roberto Álvarez es profesor titular en el Departamento de Economía de la Universidad de Chile. Correo electrónico: robalvar@econ.uchile.cl.

Aldo González es profesor asociado en el Departamento de Economía de la Universidad de Chile. Correo electrónico: agonzalez@econ.uchile.cl.

\footnotetext{
1 Los autores agradecen la colaboración de María Isidora Palma. El trabajo contó con financiamiento de ProlMed AG.
} 


\section{Introducción}

En el presente artículo se comparan los precios de los medicamentos entre distintos países de América Latina. Los productos farmacéuticos constituyen una parte no menor del gasto en salud, siendo este un tema que ha sido analizado por organismos internacionales con el propósito de facilitar el acceso de la población a estos productos²

El análisis comparativo de precios abarca la Argentina, el Brasil, Chile. Colombia, México y el Perú, las mayores economías de la región. El ejercicio de comparación se realiza para el total de medicamentos y también a nivel desagregado por categoría. Para esto último se utilizan las clasificaciones que comúnmente se emplean en el sector: innovadores, genéricos de marca y genéricos puros. Cabe mencionar que en América Latina, a diferencia de los Estados Unidos y Europa, los medicamentos genéricos de marca o similares tienen una significativa presencia en el mercado y por ende corresponde incluirlos en el análisis como una categoría especial.

Los datos fueron proporcionados por el IMS Health (ahora IQVIA). Se cuenta con un panel de observaciones mensuales realizadas entre octubre de 2010 y noviembre de 2015 correspondientes a un total de 19.741 unidades vendidas en los seis países examinados. Gracias al empleo de un modelo econométrico de panel con efecto fijo por país y controlando las variables relacionadas con el formato de venta de los productos, obtenemos un ordenamiento o clasificación de precios entre los países. La estadística proporcionada por IMS Health permite hacer las comparaciones tanto a nivel de mercado mayorista o -salida de laboratorio- como de venta al público final.

Los trabajos de comparación de precios o marcos de referencia (benchmarking) resultan útiles para sustentar las políticas de acceso a medicamentos que implementan los países. Las economías que cuentan con mecanismos de regulación de precios de fármacos -como el Brasil y Colombia- se basan en comparaciones internacionales para establecer límites a los precios de venta. En este sentido, la contribución de este trabajo es múltiple. En primer lugar, la especificación econométrica empleada permite aprovechar el gran acopio de datos del panel en cuanto a la variedad y número de unidades vendidas en cada país y a las características de comercialización de los fármacos. En segundo lugar, la comparación se realiza en distintas etapas de la cadena productiva y también por tipo de medicamento.

A continuación se detallan los resultados. En salida de fábrica y a nivel agregado de medicamento, la clasificación de países, del más barato al más caro es la siguiente: $1^{\circ}$ Argentina, $2^{\circ}$ Perú, $3^{\circ}$ México, $4^{\circ}$ Chile, $5^{\circ}$ Colombia y $6^{\circ}$ Brasil. En los productos innovadores la Argentina sería el país más barato y el Brasil ocuparía la segunda posición. En genéricos de marca, la Argentina y luego México tendrían los menores precios. Por último, en la categoría de genéricos puros el Perú es el país más barato, seguido de Chile. Las posiciones relativas de los países no cambian sustancialmente si la comparación se hace respecto al precio al público.

La clasificación de precios presentada se debe interpretar como el efecto del país en el precio, que se obtiene a partir de la comparación entre productos lo más similares posible. Por esta razón, en el análisis comparativo se aplican controles respecto a diversos factores que, además del país de venta o fabricación, repercuten en el precio.

Las diferencias de precios observadas atribuibles a los países pueden tener múltiples causas, a saber, costos de insumos, barreras arancelaras, impuestos, regulaciones en la industria, poder de mercado de los participantes, o preferencias de los consumidores. En este trabajo solo se realiza la comparación de precios entre países, sin evaluar los factores que explicarían dichas diferencias. Es uno de los primeros en que se usa una muestra de varios países para un período de tiempo extenso.

\footnotetext{
2 De acuerdo a OCDE (2011) el gasto en medicamentos constituye, en promedio, el 19\% del gasto total en salud en países miembros de dicha Organización.
} 
Además, en las comparaciones se controla un amplio grupo de variables referentes a las características de los medicamentos.

El trabajo se estructura de la siguiente forma: en la segunda sección se describen los datos empleados para el análisis. En la tercera sección se señala la metodología econométrica. En la cuarta sección se presentan los resultados, tanto por tipo de fármaco como por etapa dentro de la cadena de valor. Por último, en la quinta sección figuran las conclusiones.

\section{Datos}

Los datos de precios y otras características de los productos fueron proporcionados por IMS Health, empresa especializada en la recolección de información relevante en el sector de la salud a nivel mundial y principal fuente de datos para realizar estudios de precios de medicamentos, así como para la gestión comercial de los actores del mercado. El período estudiado abarca los 60 meses transcurridos entre octubre de 2010 y noviembre de 2015.

La comparación se realizó seleccionando el $80 \%$ de moléculas sólidas orales más vendidas en Chile. La muestra contiene 118 moléculas distintas, de las cuales 103 estuvieron presentes en los seis países durante algún mes del período estudiado. Por molécula se entiende un compuesto que tiene asociado un principio activo, el cual es comercializado bajo un nombre comercial o en forma genérica, con diversos formatos de presentación.

Los datos de precios de IMS Health se recogen en distintas partes de la cadena de producción en los países analizados. Es preciso hacer una comparación adecuada con los precios en una misma etapa a nivel vertical. Para obtener los precios, tanto a nivel minorista como a salida de fábrica, se utilizaron los factores recomendados por IMS Health para cada país, los que se consideran invariantes en el tiempo ${ }^{3}$. Cabe mencionar que para el mercado minorista se obtiene información a nivel de farmacias, lo cual no incluye las compras realizadas y asignadas a pacientes mediante el sistema público de salud.

Para el mercado chileno se usaron factores diferenciados para medicamentos de mercado ético o de venta con receta, popular y genérico y, en el caso de los medicamentos de venta directa, a través de alguna de las tres cadenas de farmacias y de venta indirecta (por medio de farmacias independientes). En el cuadro 1 se presentan los factores utilizados en cada país para obtener el precio de venta a público y el de salida de fábrica.

Para la aplicación de cada factor se cuenta con el detalle de ventas directas e indirectas para cada medicamento al mes de octubre de 2015. Con base a este, se calculó para cada producto el porcentaje que se vendía a través de cada canal y se aplicó este porcentaje para todos los períodos. Se da por sentado que para cada producto el porcentaje vendido a través de cada canal no varía a través del tiempo.

Respecto a los datos de cantidad, la información corresponde a unidades normales que representan el número de envases vendidos en el mes, y unidades estándar, que representan la cantidad de comprimidos. Para fines de este estudio, se calculan los precios minoristas unitarios, dividiendo el precio en dólares en unidades estándar.

\footnotetext{
3 Los factores recomendados por IMS Health, estimados en base a su conocimiento de los mercados locales tanto mayoristas como minoristas y los márgenes que los distintos segmentos aplican, constituyen un sustituto no perfecto pero útil de la medición en la misma etapa de la cadena de producción.
} 
Cuadro 1

América Latina (6 países): factores de conversión de precios

\begin{tabular}{|c|c|c|c|}
\hline País & \multicolumn{2}{|c|}{$\begin{array}{c}\text { Factor } \\
\text { Precio al público }\end{array}$} & $\begin{array}{c}\text { Factor } \\
\text { Precio de fábrica }\end{array}$ \\
\hline Argentina & \multicolumn{2}{|c|}{1,5125} & 0,87 \\
\hline Brasil & \multicolumn{2}{|c|}{1,3574} & 0,85 \\
\hline Colombia & \multicolumn{2}{|c|}{1,3300} & 0,92 \\
\hline México & \multicolumn{2}{|c|}{1,2150} & 0,84 \\
\hline \multirow[t]{2}{*}{ Perú } & \multicolumn{2}{|c|}{1,2000} & 0,89 \\
\hline & Venta directa & Venta indirecta & \\
\hline Chile & & & 1,00 \\
\hline Ético & 1,428 & 1,671 & \\
\hline Popular & 1,618 & 1,733 & \\
\hline Genérico & 1,761 & 1,779 & \\
\hline
\end{tabular}

Fuente: Elaboración propia, sobre la base de datos de IMS Health.

La base de datos contiene información de una serie de variables referentes a las características de los productos y que pueden afectar su precio de venta. El uso de estas variables permite aislar el factor país de otros factores que puedan incidir en el precio de los fármacos. Las características consideradas son las siguientes:

Formato: el principio activo del medicamento puede presentarse como grageas, cápsulas, especial, tabletas, polvo y ungüento. La muestra total incluye solamente las formas sólidas. En el cuadro 2 se muestra su distribución por países. En general, y para todos los países, salvo el Brasil, las tabletas representan un porcentaje mayoritario de los productos analizados.

Cuadro 2

América Latina (6 países): formato por país, muestra total

\begin{tabular}{lrrrrrrr}
\hline & Argentina & Brasil & Chile & Colombia & México & Perú & Total \\
\hline Cápsulas & 547 & 783 & 382 & 625 & 931 & 330 & 3598 \\
\hline Especial & 0 & 2 & 1 & 1 & 0 & 1 & 5 \\
\hline Grageas & 1739 & 2056 & 873 & 738 & 459 & 722 & 6587 \\
\hline Tabletas & 2077 & 1633 & 895 & 1585 & 2485 & 876 & 9551 \\
\hline Total & 4363 & 4474 & 2151 & 2949 & 3875 & 1929 & 19741 \\
\hline
\end{tabular}

Fuente: Elaboración propia, sobre la base de datos de IMS Health.

En cuanto a la clasificación por tipo de medicamento, la información provista por IMS Health permite clasificar los fármacos en innovadores, similares y genéricos. Los primeros corresponden a aquellos productos denominados pioneros, que fueron lanzados al mercado bajo protección de patente, independientemente de si al día de hoy la patente está vigente o no. Los productos denominados similares o genéricos de marca poseen el mismo principio activo que los pioneros, pero se comercializan bajo una marca comercial. Por último, los medicamentos genéricos se venden bajo el nombre del compuesto o principio activo que este contiene.

En la base de datos original existen genéricos, similares y de marca. De acuerdo a IMS Health, la categoría marca contiene tanto similares como innovaciones. Para diferenciar entre ambos tipos de fármacos dividimos la categoría marca utilizando el criterio de que los productos de marca de laboratorios nacionales serían clasificados como similares y los de laboratorios multinacionales como innovaciones. Se ha procedido de esta manera porque los datos con los que contamos no nos permiten identificar a los laboratorios nacionales que poseen licencias, motivo por el cual usamos una 
aproximación. De acuerdo a información suministrada por IMS Health, el 98\% de las innovaciones provienen de laboratorios multinacionales ${ }^{4}$. En el cuadro 3 se muestra la distribución por tipo de medicamentos. Se puede apreciar que en la muestra general, y en todos los países, predominan los productos clasificados como similares.

\section{Cuadro 3}

América Latina (6 países): tipos de medicamento por país, muestra total

\begin{tabular}{lrrrrrrr}
\hline & Argentina & Brasil & Chile & Colombia & México & Perú & Total \\
\hline Innovador & 654 & 449 & 426 & 828 & 823 & 388 & 3568 \\
\hline Genérico de marca & 3200 & 2299 & 1592 & 1153 & 1768 & 1096 & 11108 \\
\hline Genérico & 509 & 1726 & 133 & 968 & 1284 & 445 & 5065 \\
\hline Total & 4363 & 4474 & 2151 & 2949 & 3875 & 1929 & 19741 \\
\hline
\end{tabular}

Fuente: Elaboración propia, sobre la base de datos de IMS Health.

Los medicamentos vienen en distintas presentaciones, factor que podría influir en las diferencias de precio de un mismo fármaco. La muestra incluye 1.114 tipos de envases; como ejemplo se podría citar el paquete de $10 \mathrm{mg} \times 30$. En el cuadro 4 se indica el número de observaciones, de moléculas distintas y de envases distintos por país. Se aprecia que existe en todos los países una gran variedad en esta dimensión.

\section{Cuadro 4}

América Latina (6 países): tipos de envases

\begin{tabular}{lccc}
\hline & Observaciones & Moléculas & Envases \\
\hline Argentina & 4363 & 114 & 492 \\
\hline Brasil & 4474 & 109 & 581 \\
\hline Chile & 2151 & 117 & 401 \\
\hline Colombia & 2949 & 112 & 467 \\
\hline México & 3875 & 112 & 489 \\
\hline Perú & 1929 & 110 & 371 \\
\hline Total & 19741 & 674 & 2801 \\
\hline
\end{tabular}

Fuente: Elaboración propia, sobre la base de datos de IMS Health.

En el cuadro 5 se muestra el número de coincidencias de tipo de envases entre países. Los números indican cuántos envases se repiten entre cada país y la diagonal muestra el total de envases distintos que hay en cada país. Se puede apreciar que existe un grado importante de coincidencia, lo que resulta útil a fin de comparar los precios entre los países. Por ejemplo, de los 492 tipos de envases que existen en la Argentina, más de la mitad también se encuentran disponibles en los demás países.

Cuadro 5

América Latina (6 países): coincidencias de envases entre países, muestra total

\begin{tabular}{lcccccc}
\hline & Argentina & Brasil & Chile & Colombia & México & Perú \\
\hline Argentina & 492 & 291 & 249 & 266 & 278 & 239 \\
\hline Brasil & & 581 & 244 & 286 & 275 & 233 \\
\hline Chile & & & 401 & 226 & 236 & 203 \\
\hline Colombia & & & & 467 & 269 & 238 \\
\hline México & & & & & 489 & 236 \\
\hline Perú & & & & & & 371 \\
\hline
\end{tabular}

Fuente: Elaboración propia, sobre la base de datos de IMS Health.

${ }^{4}$ La lista de laboratorios multinacionales y nacionales también fue proporcionada por IMS Health. 


\section{Metodología}

Una de las principales deficiencias de la simple comparación de precios de medicamentos es que estos últimos presentan características muy heterogéneas. Esta situación se complica aún más si se pretende comparar los precios entre los países y a lo largo del tiempo. La literatura especializada indica que los precios de los productos pueden diferir entre los países por diversas razones: por ejemplo, debido a diferencias en la unidad de medida o el tipo de envase (Cameron y otros, 2009; Danzon y Furukawa, 2011).

Para ello es necesario controlar las variables del mayor número de características, a fin de lograr una comparación más adecuada. En nuestro caso ello es posible porque se dispone de abundante información sobre las características de los medicamentos descritos en la sección anterior. Para analizar si existen diferencias de precios entre países para productos similares, se ha de emplear la siguiente ecuación:

$$
\log P_{m c t}=\delta_{c}+\delta_{t}+\sum_{k=1}^{K} \beta_{k} X_{k m}+\varepsilon_{m c t}
$$

donde $P$ es el precio de la molécula ${ }^{5}, c$ denota el país y $t$ el tiempo. $\delta_{c}$ es un efecto fijo por país que captura las diferencias de precios entre países y se define como una variable categórica igual a $1 \mathrm{si}$ la molécula es vendida en el país $c$ y 0 en caso contrario. Estas diferencias de precios son las que se obtienen luego de controlar las variables de efectos temporales $\left(\delta_{t}\right)$ y las referentes a las características de las moléculas (resumidas en el vector $X$ ).

El vector $X$ está compuesto por una serie de variables categóricas definidas por molécula (más de 100 moléculas distintas por país), formato (grageas, cápsulas, especial y tabletas), efecto del medicamento (que puede ser inmediato o retardado), paquete (más de 1.000 variedades de envases) y tipo (innovadores, similares y genéricos). En todos los casos, se excluye una categoría base de la estimación. Dada la gran cantidad de parámetros asociados a estas variables categóricas, estos no figuran en los cuadros de resultados.

Las diferencias de precios capturadas por el efecto fijo país resumen todo aquello que incide en los precios y que varía entre los distintos países. Como ejemplo cabe citar las diferencias en los márgenes, impuestos y preferencias, que se asume no cambian a lo largo del tiempo. En el caso de este estudio, dado el breve período de análisis, se estima que se trata de un supuesto razonable ${ }^{6}$.

El efecto fijo por país se interpreta como la diferencia de precios en términos porcentuales -ya que el precio se expresa en logaritmo- del país $c$ en comparación con aquel que se define como referencia, que en nuestras estimaciones es la Argentina?.

Los cuadros 6A y 6B presentan la estadística descriptiva de los precios (logaritmo de precios en dólares) utilizados en las estimaciones, así como el número de observaciones disponibles. Tanto para el precio al público como para el precio de salida de fábrica el promedio más alto se encuentra en Chile, solo superado por Colombia. En el gráfico 1 y 2 se muestra la evolución de los precios promedio en el tiempo para ambos precios utilizados, donde se puede apreciar una tendencia a la baja junto con una convergencia hacia menores diferencias al final del período analizado.

\footnotetext{
5 Los precios se expresan en dólares para que estén en una unidad común.

6 Se revisó si en estos países hubo cambios regulatorios sustantivos y no se encontró evidencia que ello hubiese ocurrido.

7 Se eligió la Argentina por razón de orden alfabético. Cabe señalar que la elección del país base no cambia los resultados de la estimación: solo cambia la interpretación del parámetro.
} 


\section{Cuadro 6}

América Latina (6 países): estadística descriptiva del logaritmo del precio unitario (En logaritmo de precios en dólares)

\section{A. Al público}

\begin{tabular}{lccccc}
\hline País & Observaciones & Media & $\begin{array}{c}\text { Desviación } \\
\text { estándar }\end{array}$ & Mínimo & Máximo \\
\hline Argentina & 192676 & 1,42 & 3,97 & 0 & 301,7 \\
\hline Brasil & 181698 & 1,76 & 3,73 & 0,0034 & 149,3 \\
\hline Chile & 87802 & 1,88 & 4,21 & 0,006 & 111,4 \\
\hline Colombia & 123974 & 2,75 & 6,85 & 0,006 & 144,4 \\
\hline México & 131903 & 1,66 & 4,23 & $7,59 \times 10^{-6}$ & 162,7 \\
\hline Perú & 75879 & 1,58 & 4,95 & 0,0000286 & 173,1 \\
\hline
\end{tabular}

Fuente: Elaboración propia, sobre la base de datos de IMS Health.

\section{B. Salida de fábrica}

\begin{tabular}{lccccc}
\hline País & Observaciones & Media & $\begin{array}{c}\text { Desviación } \\
\text { estándar }\end{array}$ & Mínimo & Máximo \\
\hline Argentina & 192676 & $-0,88$ & 1,07 & $-9,58$ & 5,16 \\
\hline Brasil & 181698 & $-0,61$ & 1,13 & $-6,16$ & 4,54 \\
\hline Chile & 92551 & $-0,49$ & 1,195 & $-5,69$ & 4,35 \\
\hline Colombia & 123974 & $-0,46$ & 1,61 & $-5,48$ & 4,60 \\
\hline México & 131903 & $-0,87$ & 1,57 & $-12,16$ & 4,72 \\
\hline Perú & 75879 & $-0,87$ & 1,52 & $-10,76$ & 4,85 \\
\hline
\end{tabular}

Fuente: Elaboración propia, sobre la base de datos de IMS Health.

\section{Gráfico 1}

América Latina (6 países): evolución de los precios de los medicamentos, precio al público, octubre de 2010 a noviembre de 2015

(Logaritmo del precio unitario en dólares)

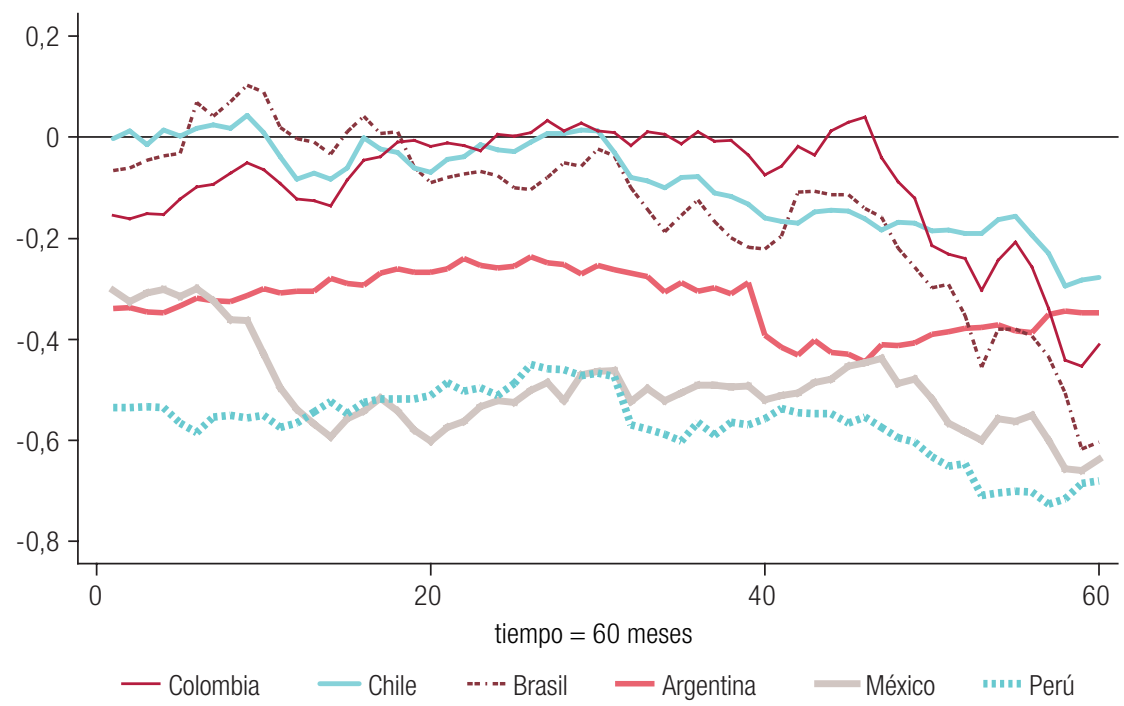

Fuente: Elaboración propia, sobre la base de datos de IMS Health. 


\section{Gráfico 2}

América Latina (6 países): evolución de los precios de los medicamentos, salida de fábrica, octubre de 2010 a noviembre de 2015

(Logaritmo del precio unitario en dólares)

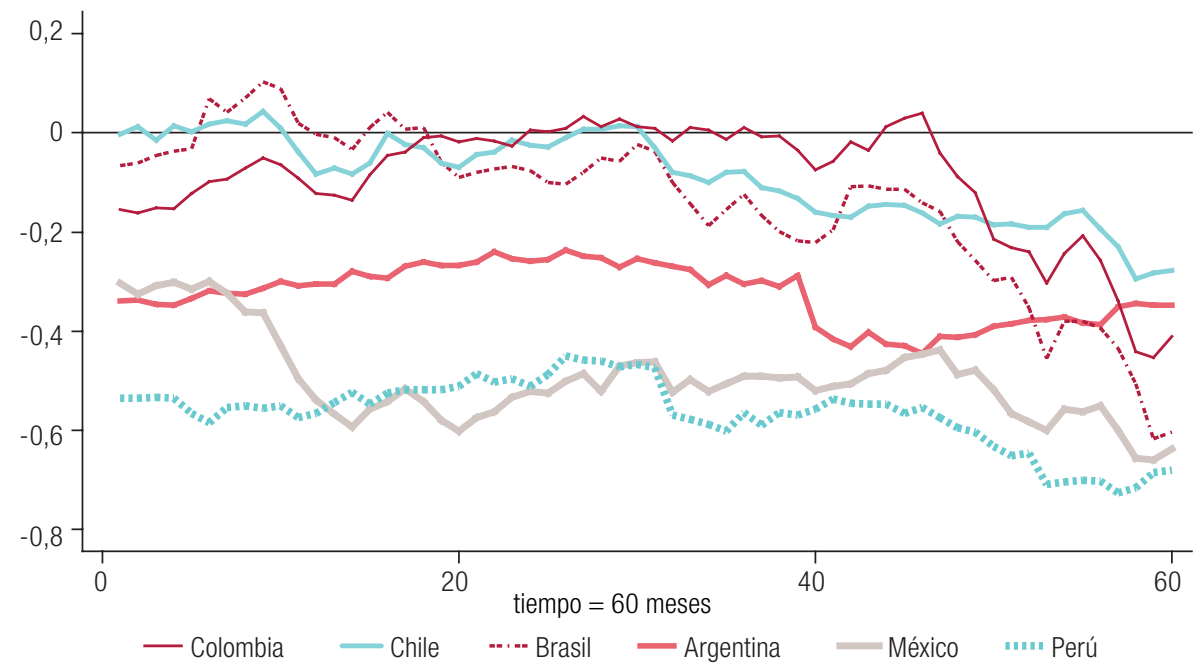

Fuente: Elaboración propia, sobre la base de datos de IMS Health.

\section{Resultados}

La ecuación de precios se estimó para toda la muestra en dos puntos de la cadena, la salida de fábrica y el precio al público, así como para los tres tipos de medicamentos mencionados. Los resultados econométricos para la muestra total figuran en el cuadro 7 para los precios de fábrica y en el cuadro 8 para los precios al público. En las columnas (1) a (5) se introducen las variables de control referentes a las características de los productos. La columna (5) incluye a todas estas últimas.

En el caso de los precios en salida de fábrica (cuadro 7), se aprecia que en Chile los precios de los medicamentos en su conjunto son superiores a los vigentes en la Argentina, con una diferencia cercana al 20\% y estable en el tiempo. Los precios en Chile son superiores también a los de México y el Perú, pero inferiores a los de Colombia y el Brasil. En los precios al público final (cuadro 8), los resultados son bastante similares. Chile no tiene precios estadísticamente distintos de la Argentina, que es el país base, pero sí más altos que los precios de los medicamentos en México y el Perú. Por el contrario, los precios en Chile serían inferiores a los vigentes en Colombia y el Brasil.

Se aplicó el test de Fisher para evaluar si las diferencias en las posiciones en la clasificación son estadísticamente significativas. Los resultados señalan que en la muestra total para los precios de salida de fábrica Chile no es estadísticamente más caro o barato que México y el Perú (véase el anexo A1). Respecto a los precios al público en la muestra total la posición de Chile no diferiría de la de Colombia y México.

En los cuadros 9 a 14 se presentan los resultados por tipo de medicamentos, desglosados en innovadores, genéricos de marca y genéricos puros. En el caso de los originarios, las estimaciones indican que la Argentina tiene los menores precios, a bastante distancia de los países que lo siguen: el Brasil y Chile en salida de fábrica, y el Perú y Chile en precio al público final. En los fármacos similares, la Argentina y México presentan los menores precios promedio tanto en mercado mayorista y minorista, aunque en este último segmento los precios en México se sitúan un 12\% por debajo de los de la Argentina. Finalmente en los medicamentos genéricos, Perú es el país más barato en precios al público final, seguido de Chile, y ambos países compartirían el primer lugar de menores precios en la comparación a salida de laboratorio. 


\section{Cuadro 7}

América Latina (6 países): precios de fábrica a nivel agregado

\begin{tabular}{|c|c|c|c|c|c|}
\hline Variables & (1) & (2) & (3) & (4) & (5) \\
\hline \multirow{2}{*}{ Brasil } & $0,267^{* \star \star}$ & $0,359^{* \star *}$ & $0,368^{* \star *}$ & $0,323^{\text {***}}$ & $0,539^{\star \star \star *}$ \\
\hline & $(0,000870)$ & $(0,00187)$ & $(0,00478)$ & $(0,0131)$ & $(0,0940)$ \\
\hline \multirow{2}{*}{ Chile } & $0,387^{* \star *}$ & $0,255^{\star \star \star}$ & $0,255^{\star \star \star}$ & $0,237^{\star \star \star *}$ & $0,201^{\text {***}}$ \\
\hline & $(0,000482)$ & $(0,0205)$ & $(0,0217)$ & $(0,0259)$ & $(0,0219)$ \\
\hline \multirow{2}{*}{ Colombia } & $0,419^{\star * \star}$ & $0,371^{* \star *}$ & $0,392^{* \star *}$ & $0,394^{* \star *}$ & $0,451^{\star \star \star *}$ \\
\hline & $(0,000370)$ & $(0,0171)$ & $(0,0211)$ & $(0,0232)$ & $(0,0807)$ \\
\hline \multirow{2}{*}{ México } & $0,0107^{\star \star \star}$ & $0,112^{\star \star}$ & $0,157^{\star \star}$ & $0,123^{*}$ & $0,148^{*}$ \\
\hline & $(0,00152)$ & $(0,0417)$ & $(0,0503)$ & $(0,0559)$ & $(0,0695)$ \\
\hline \multirow{2}{*}{ Perú } & $0,0140^{\star * \star}$ & $-0,0692^{\star \star}$ & $-0,0585^{\star \star}$ & 0,104 & 0,111 \\
\hline & $(0,000457)$ & $(0,0172)$ & $(0,0170)$ & $(0,0535)$ & $(0,0567)$ \\
\hline \multirow{2}{*}{ Constante } & $-0,836^{\star \star *}$ & $-1,115^{\star \star}$ & $-0,946^{\star *}$ & $-0,700^{\star *}$ & $-1,379^{\star *}$ \\
\hline & $(0,0353)$ & $(0,291)$ & $(0,321)$ & $(0,261)$ & $(0,375)$ \\
\hline Observaciones & 798681 & 798681 & 798681 & 798681 & 798681 \\
\hline R-cuadrado & 0,021 & 0,554 & 0,568 & 0,680 & 0,751 \\
\hline Tiempo & Sí & Sí & Sí & Sí & Sí \\
\hline Moléculas & NO & SÍ & Sí & Sí & SÍ \\
\hline Formato & NO & NO & Sí & Sí & Sí \\
\hline Efecto & NO & NO & Sí & Sí & Sí \\
\hline Paquete & NO & NO & NO & Sí & Sí \\
\hline Tipo & NO & NO & NO & NO & Sí \\
\hline
\end{tabular}

Fuente: Elaboración propia, sobre la base de datos de IMS Health.

Nota: Los errores estándar figuran entre paréntesis ${ }^{\star \star \star} p<0,01,{ }^{\star \star} p<0,05,{ }^{*} p<0,1$.

\section{Cuadro 8}

América Latina (6 países): precios al público a nivel agregado

\begin{tabular}{|c|c|c|c|c|c|}
\hline Variables & (1) & (2) & (3) & (4) & (5) \\
\hline \multirow{2}{*}{ Brasil } & $0,182^{\star \star \star}$ & $0,272^{\star \star \star}$ & $0,281^{\star \star \star}$ & $0,238^{\star \star \star}$ & $0,451^{\star \star \star}$ \\
\hline & $(0,000877)$ & $(0,00137)$ & $(0,00524)$ & $(0,0133)$ & $(0,0930)$ \\
\hline \multirow{2}{*}{ Chile } & $0,236^{\star \star \star}$ & $0,0966^{\star \star \star}$ & $0,0959^{\star \star \star}$ & $0,0724^{\star *}$ & 0,0369 \\
\hline & $(0,00127)$ & $(0,0220)$ & $(0,0234)$ & $(0,0259)$ & $(0,0216)$ \\
\hline \multirow{2}{*}{ Colombia } & $0,235^{\star \star \star}$ & $0,186^{\star \star \star}$ & $0,206^{\star \star \star}$ & $0,209^{\star \star \star}$ & $0,265^{\star *}$ \\
\hline & $(0,000372)$ & $(0,0163)$ & $(0,0205)$ & $(0,0233)$ & $(0,0802)$ \\
\hline \multirow{2}{*}{ México } & $-0,173^{\star \star \star}$ & $-0,0725$ & $-0,0277$ & $-0,0620$ & $-0,0384$ \\
\hline & $(0,00154)$ & $(0,0419)$ & $(0,0504)$ & $(0,0562)$ & $(0,0693)$ \\
\hline \multirow{2}{*}{ Perú } & $-0,240^{\star \star \star}$ & $-0,323^{\star \star \star}$ & $-0,312^{\star \star \star}$ & $-0,150^{\star \star}$ & $-0,145^{\star}$ \\
\hline & $(0,000457)$ & $(0,0175)$ & $(0,0173)$ & $(0,0540)$ & $(0,0567)$ \\
\hline \multirow{2}{*}{ Constante } & $-0,281^{\star \star \star}$ & $-0,571$ & $-0,402$ & $-0,167$ & $-0,837^{*}$ \\
\hline & $(0,0360)$ & $(0,283)$ & $(0,314)$ & $(0,255)$ & $(0,372)$ \\
\hline Observaciones & 793932 & 793932 & 793932 & 793932 & 793932 \\
\hline R-cuadrado & 0,021 & 0,555 & 0,569 & 0,680 & 0,751 \\
\hline Tiempo & Sí & Sí & Sí & Sí & Sí \\
\hline Moléculas & NO & Sí & SÍ & SÍ & Sí \\
\hline Formato & NO & NO & Sí & Sí & Sí \\
\hline Efecto & NO & NO & Sí & Sí & Sí \\
\hline Paquete & NO & NO & NO & Sí & Sí \\
\hline Tipo & NO & NO & NO & NO & Sí \\
\hline
\end{tabular}

Fuente: Elaboración propia, sobre la base de datos de IMS Health.

Nota: Los errores estándar figuran entre paréntesis ${ }^{* \star} p<0,01,{ }^{* \star} p<0,05,{ }^{*} p<0,1$. 


\section{Cuadro 9}

América Latina (6 países): precios de fábrica de medicamentos innovadores

\begin{tabular}{|c|c|c|c|c|}
\hline Variables & (1) & (2) & (3) & (4) \\
\hline \multirow{2}{*}{ Brasil } & $0,695^{\star \star \star}$ & $0,595^{\star \star \star}$ & $0,580^{\text {***}}$ & $0,534^{\star * \star}$ \\
\hline & $(0,000681)$ & $(0,0240)$ & $(0,0232)$ & $(0,0254)$ \\
\hline \multirow{2}{*}{ Chile } & $0,787^{\star \star \star}$ & $0,685^{\text {***}}$ & $0,675^{\text {***}}$ & $0,625^{\star \star \star}$ \\
\hline & $(0,000932)$ & $(0,0331)$ & $(0,0314)$ & $(0,0346)$ \\
\hline \multirow{2}{*}{ Colombia } & $1,091^{\star \star \star}$ & $1,087^{\star \star \star}$ & $1,101^{\text {***}}$ & $1,034^{* * *}$ \\
\hline & $(0,000915)$ & $(0,0306)$ & $(0,0299)$ & $(0,0283)$ \\
\hline \multirow{2}{*}{ México } & $0,781^{\star \star *}$ & $0,750^{\text {***}}$ & $0,768^{\star \star \star}$ & $0,717^{\text {***}}$ \\
\hline & $(0,00129)$ & $(0,0314)$ & $(0,0327)$ & $(0,0279)$ \\
\hline \multirow{2}{*}{ Perú } & $0,738^{\star \star *}$ & $0,679^{\star \star *}$ & $0,678^{\star * *}$ & $0,644^{* * *}$ \\
\hline & $(0,00172)$ & $(0,0265)$ & $(0,0248)$ & $(0,0239)$ \\
\hline \multirow{2}{*}{ Constante } & $-0,633^{\star \star *}$ & $-1,314^{* *}$ & $-1,330^{\star *}$ & $-0,843^{\star *}$ \\
\hline & $(0,0269)$ & $(0,365)$ & $(0,360)$ & $(0,223)$ \\
\hline Observaciones & 161925 & 161925 & 161925 & 161925 \\
\hline R-cuadrado & 0,088 & 0,772 & 0,783 & 0,875 \\
\hline Tiempo & Sí & Sí & Sí & Sí \\
\hline Moléculas & NO & Sí & Sí & SÍ \\
\hline Formato & NO & NO & Sí & Sí \\
\hline Efecto & NO & NO & Sí & Sí \\
\hline Paquete & NO & NO & NO & Sí \\
\hline
\end{tabular}

Fuente: Elaboración propia, sobre la base de datos de IMS Health.

Nota: Los errores estándar figuran entre paréntesis ${ }^{* \star *} p<0,01,{ }^{* *} p<0,05,{ }^{*} p<0,1$.

Cuadro 10

América Latina (6 países): precios al público de medicamentos innovadores

\begin{tabular}{|c|c|c|c|c|}
\hline Variables & (1) & (2) & (3) & (4) \\
\hline \multirow{2}{*}{ Brasil } & $0,610^{\star \star \star}$ & $0,510^{\star \star \star}$ & $0,495^{\star \star \star}$ & $0,448^{\star \star \star}$ \\
\hline & $(0,000682)$ & $(0,0240)$ & $(0,0232)$ & $(0,0258)$ \\
\hline \multirow{2}{*}{ Chile } & $0,619^{\star \star \star}$ & $0,503^{\star \star \star}$ & $0,492^{\star \star \star}$ & $0,440^{\star \star \star}$ \\
\hline & $(0,000370)$ & $(0,0336)$ & $(0,0318)$ & $(0,0351)$ \\
\hline \multirow{2}{*}{ Colombia } & $0,906^{\star \star \star}$ & $0,903^{\star \star \star}$ & $0,917^{\star \star \star}$ & $0,850^{\star \star \star}$ \\
\hline & $(0,000915)$ & $(0,0305)$ & $(0,0299)$ & $(0,0283)$ \\
\hline \multirow{2}{*}{ México } & $0,597^{\star \star \star}$ & $0,566^{\star \star \star}$ & $0,584^{\star \star \star}$ & $0,533^{\star \star \star}$ \\
\hline & $(0,00129)$ & $(0,0313)$ & $(0,0327)$ & $(0,0282)$ \\
\hline \multirow{2}{*}{ Perú } & $0,484^{\star \star \star}$ & $0,425^{\star \star \star}$ & $0,423^{\star \star \star}$ & $0,389^{\star \star \star}$ \\
\hline & $(0,00172)$ & $(0,0265)$ & $(0,0248)$ & $(0,0238)$ \\
\hline \multirow{2}{*}{ Constante } & $-0,0767^{* *}$ & $-0,765^{*}$ & $-0,780^{*}$ & $-0,294$ \\
\hline & $(0,0274)$ & $(0,362)$ & $(0,358)$ & $(0,225)$ \\
\hline Observaciones & 161218 & 161218 & 161218 & 161218 \\
\hline R-cuadrado & 0,063 & 0,765 & 0,776 & 0,871 \\
\hline Tiempo & Sí & sí & Sí & Sí \\
\hline Moléculas & NO & sí & sí & sí \\
\hline Formato & NO & NO & Sí & sí \\
\hline Efecto & NO & NO & Sí & sí \\
\hline Paquete & NO & NO & NO & Sí \\
\hline
\end{tabular}

Fuente: Elaboración propia, sobre la base de datos de IMS Health.

Nota: Los errores estándar figuran entre paréntesis ${ }^{\star \star \star} p<0,01$, ${ }^{\star \star} p<0,05,{ }^{*} p<0,1$. 


\section{Cuadro 11}

América Latina (6 países): precios de fábrica de los medicamentos similares

\begin{tabular}{|c|c|c|c|c|}
\hline Variables & (1) & (2) & (3) & (4) \\
\hline \multirow{2}{*}{ Brasil } & $0,0768^{* * *}$ & $0,278^{\star \star \star}$ & $0,290^{\star \star \star}$ & $0,240^{\star \star * *}$ \\
\hline & $(0,000242)$ & $(0,0164)$ & $(0,0154)$ & $(0,00952)$ \\
\hline \multirow{2}{*}{ Chile } & $0,366^{\star \star \star}$ & $0,266^{\star \star \star}$ & $0,265^{\star \star \star}$ & $0,259^{* * *}$ \\
\hline & $(0,000664)$ & $(0,0185)$ & $(0,0191)$ & $(0,0363)$ \\
\hline \multirow{2}{*}{ Colombia } & $0,847^{\star \star \star}$ & $0,725^{\text {***}}$ & $0,717^{\star \star \star *}$ & $0,681^{\star \star *}$ \\
\hline & $(0,00144)$ & $(0,0313)$ & $(0,0375)$ & $(0,0303)$ \\
\hline \multirow{2}{*}{ México } & $0,00591^{* *}$ & $0,0917^{\star}$ & $0,0997^{\star *}$ & $0,0678^{*}$ \\
\hline & $(0,00201)$ & $(0,0378)$ & $(0,0385)$ & $(0,0272)$ \\
\hline \multirow{2}{*}{ Perú } & $0,363^{\star \star *}$ & $0,242^{* \star *}$ & $0,244^{\star \star \star}$ & $0,326^{\star * *}$ \\
\hline & $(0,00110)$ & $(0,0161)$ & $(0,0181)$ & $(0,0228)$ \\
\hline \multirow{2}{*}{ Constante } & $-0,839^{* \star *}$ & $-0,969^{\star \star *}$ & $-0,859^{\star * *}$ & $-0,464^{\star \star}$ \\
\hline & $(0,0198)$ & $(0,189)$ & $(0,204)$ & $(0,173)$ \\
\hline Observaciones & 447017 & 447017 & 447017 & 447017 \\
\hline R-cuadrado & 0,056 & 0,628 & 0,635 & 0,748 \\
\hline Tiempo & Sí & Sí & Sí & Sí \\
\hline Moléculas & NO & Sí & SÍ & Sí \\
\hline Formato & NO & NO & Sí & Sí \\
\hline Efecto & NO & NO & Sí & Sí \\
\hline Paquete & NO & NO & NO & Sí \\
\hline
\end{tabular}

Fuente: Elaboración propia, sobre la base de datos de IMS Health.

Nota: Los errores estándar figuran entre paréntesis ${ }^{* \star *} p<0,01,{ }^{* \star} p<0,05,{ }^{*} p<0,1$.

\section{Cuadro 12}

América Latina (6 países): precios al público de los medicamentos similares

\begin{tabular}{|c|c|c|c|c|}
\hline Variables & (1) & (2) & (3) & (4) \\
\hline \multirow{2}{*}{ Brasil } & $0,00817^{\star \star \star}$ & $0,190^{\star \star \star}$ & $0,202^{\star \star \star}$ & $0,155^{\star \star \star}$ \\
\hline & $(0,000246)$ & $(0,0156)$ & $(0,0146)$ & $(0,0102)$ \\
\hline \multirow{2}{*}{ Chile } & $0,205^{\star \star \star}$ & $0,0999^{\star \star *}$ & $0,0991^{* * *}$ & $0,0867^{\star}$ \\
\hline & $(0,00160)$ & $(0,0179)$ & $(0,0188)$ & $(0,0374)$ \\
\hline \multirow{2}{*}{ Colombia } & $0,662^{\star \star \star}$ & $0,539^{\star \star \star}$ & $0,532^{\star \star \star}$ & $0,497^{\star * *}$ \\
\hline & $(0,00146)$ & $(0,0305)$ & $(0,0373)$ & $(0,0307)$ \\
\hline \multirow{2}{*}{ México } & $-0,178^{\star \star \star}$ & $-0,0926^{\star}$ & $-0,0841^{*}$ & $-0,117^{\star \star \star}$ \\
\hline & $(0,00203)$ & $(0,0378)$ & $(0,0386)$ & $(0,0272)$ \\
\hline \multirow{2}{*}{ Perú } & $0,109^{\star \star \star}$ & $-0,0113$ & $-0,00915$ & $0,0703^{* *}$ \\
\hline & $(0,00110)$ & $(0,0167)$ & $(0,0188)$ & $(0,0231)$ \\
\hline \multirow{2}{*}{ Constante } & $-0,285^{\star \star \star}$ & $-0,421^{*}$ & $-0,313$ & 0,0700 \\
\hline & $(0,0210)$ & $(0,186)$ & $(0,203)$ & $(0,184)$ \\
\hline Observaciones & 443149 & 443149 & 443149 & 443149 \\
\hline R-cuadrado & 0,042 & 0,623 & 0,630 & 0,744 \\
\hline Tiempo & Sí & Sí & Sí & Sí \\
\hline Moléculas & NO & Sí & Sí & Sí \\
\hline Formato & NO & NO & Sí & Sí \\
\hline Efecto & NO & NO & Sí & Sí \\
\hline Paquete & NO & NO & NO & Sí \\
\hline
\end{tabular}

Fuente: Elaboración propia, sobre la base de datos de IMS Health.

Nota: Los errores estándar figuran entre paréntesis ${ }^{* \star *} p<0,01,{ }^{* \star} p<0,05,{ }^{*} p<0,1$. 
Cuadro 13

América Latina (6 países): precios de fábrica de los medicamentos genéricos

\begin{tabular}{|c|c|c|c|c|}
\hline Variables & (1) & (2) & (3) & (4) \\
\hline \multirow{2}{*}{ Brasil } & $0,808^{* \star *}$ & $0,450^{\text {***}}$ & $0,440^{\text {***}}$ & $0,456^{\star * *}$ \\
\hline & $(0,00392)$ & $(0,0655)$ & $(0,0697)$ & $(0,0272)$ \\
\hline \multirow{2}{*}{ Chile } & $-1,181^{\star \star \star}$ & $-1,164^{\star \star \star}$ & $-1,168^{\star \star \star}$ & $-1,236^{\star \star *}$ \\
\hline & $(0,00332)$ & $(0,0181)$ & $(0,0212)$ & $(0,0357)$ \\
\hline \multirow{2}{*}{ Colombia } & $-0,382^{\star \star \star}$ & $-0,650^{\text {*** }}$ & $-0,663^{\star \star \star}$ & $-0,671^{\star \star *}$ \\
\hline & $(0,00107)$ & $(0,0412)$ & $(0,0424)$ & $(0,0316)$ \\
\hline \multirow{2}{*}{ México } & $-0,432^{\star \star \star}$ & $-0,569^{\star \star *}$ & $-0,571^{\star \star \star}$ & $-0,587^{\star \star \star}$ \\
\hline & $(0,00518)$ & $(0,0173)$ & $(0,0215)$ & $(0,0342)$ \\
\hline \multirow{2}{*}{ Perú } & $-1,173^{\star \star \star}$ & $-1,449^{\star \star \star}$ & $-1,454^{\star \star \star}$ & $-1,278^{\star \star \star}$ \\
\hline & $(0,00173)$ & $(0,0286)$ & $(0,0313)$ & $(0,0621)$ \\
\hline \multirow{2}{*}{ Constante } & $-1,366^{\star \star *}$ & $-1,644^{\star * *}$ & $-1,504^{\star \star \star}$ & $-0,945^{*}$ \\
\hline & $(0,0300)$ & $(0,218)$ & $(0,142)$ & $(0,426)$ \\
\hline Observaciones & 189739 & 189739 & 189739 & 189739 \\
\hline R-cuadrado & 0,242 & 0,700 & 0,702 & 0,796 \\
\hline Tiempo & Sí & Sí & Sí & Sí \\
\hline Moléculas & NO & Sí & Sí & SÍ \\
\hline Formato & NO & NO & Sí & Sí \\
\hline Efecto & NO & NO & Sí & Sí \\
\hline Paquete & NO & NO & NO & Sí \\
\hline
\end{tabular}

Fuente: Elaboración propia, sobre la base de datos de IMS Health.

Nota: Los errores estándar figuran entre paréntesis ${ }^{\star \star *} p<0,01,{ }^{* \star} p<0,05,{ }^{*} p<0,1$.

\section{Cuadro 14}

América Latina (6 países): precios al público de los medicamentos genéricos

\begin{tabular}{|c|c|c|c|c|}
\hline Variables & (1) & (2) & (3) & (4) \\
\hline \multirow{2}{*}{ Brasil } & $0,723^{\star \star \star}$ & $0,364^{\star \star \star}$ & $0,354^{\star \star \star}$ & $0,370^{\star \star \star *}$ \\
\hline & $(0,00391)$ & $(0,0651)$ & $(0,0692)$ & $(0,0266)$ \\
\hline \multirow{2}{*}{ Chile } & $-1,160^{\text {***}}$ & $-1,161^{\text {***}}$ & $-1,165^{\star \star \star}$ & $-1,239^{* \star *}$ \\
\hline & $(0,00409)$ & $(0,0176)$ & $(0,0204)$ & $(0,0349)$ \\
\hline \multirow{2}{*}{ Colombia } & $-0,566^{\star \star \star}$ & $-0,835^{\star \star \star}$ & $-0,848^{\star \star \star}$ & $-0,857^{\star \star *}$ \\
\hline & $(0,00107)$ & $(0,0410)$ & $(0,0421)$ & $(0,0314)$ \\
\hline \multirow{2}{*}{ México } & $-0,616^{\star \star \star}$ & $-0,753^{\text {** }}$ & $-0,756^{\star \star \star}$ & $-0,774^{\star \star \star}$ \\
\hline & $(0,00517)$ & $(0,0171)$ & $(0,0208)$ & $(0,0337)$ \\
\hline \multirow{2}{*}{ Perú } & $-1,427^{\star \star *}$ & $-1,703^{\star * \star}$ & $-1,709^{\star * \star}$ & $-1,534^{\star * *}$ \\
\hline & $(0,00173)$ & $(0,0286)$ & $(0,0313)$ & $(0,0619)$ \\
\hline \multirow{2}{*}{ Constante } & $-0,812^{\star \star \star}$ & $-1,091^{\star \star \star}$ & $-0,952^{\star \star \star}$ & $-0,386$ \\
\hline & $(0,0298)$ & $(0,218)$ & $(0,141)$ & $(0,421)$ \\
\hline Observaciones & 189565 & 189565 & 189565 & 189565 \\
\hline R-cuadrado & 0,267 & 0,709 & 0,712 & 0,802 \\
\hline Tiempo & Sí & Sí & Sí & Sí \\
\hline Moléculas & NO & Sí & Sí & Sí \\
\hline Formato & NO & NO & Sí & Sí \\
\hline Efecto & NO & NO & Sí & SÍ \\
\hline Paquete & NO & NO & NO & Sí \\
\hline
\end{tabular}

Fuente: Elaboración propia, sobre la base de datos de IMS Health.

Nota: Los errores estándar figuran entre paréntesis ${ }^{\star \star *} p<0,01,{ }^{* \star} p<0,05,{ }^{*} p<0,1$. 
Explicar las causas de las diferencias de precios entre países excede el alcance de este estudio. Sin embargo, relacionaremos nuestros resultados con las políticas de control de precios de los países de la muestra. Los países que presentan los mayores precios tanto a salida de fábrica como en venta al público final -el Brasil y Colombia- son también los únicos que poseen mecanismos formales de regulación de precios de medicamentos. Si nos enfocamos en los productos originarios o innovadores, que son los que serían más susceptibles de ser regulados debido a que enfrentan una menor competencia, Colombia sería el más caro en ambas etapas de la cadenas vertical, mientras que la posición del Brasil mejora sustantivamente, siendo el segundo más barato luego de la Argentina en salida de fábrica y el tercero más caro en venta al público.

El Brasil cuenta desde 2003 con un sistema en virtud del cual el precio de los fármacos sometidos a regulación no puede ser superior al menor precio de un conjunto de países de referencia ${ }^{8}$. En Colombia se definen los medicamentos que han de ser regulados en base a consideraciones de competencia, la cual se mide en base a la concentración en el respectivo mercado. Luego se calcula el precio de referencia internacional (PRI) para cada uno de ellos en base a una muestra de 16 países, que incluye economías de la Organización de Cooperación y Desarrollo Económicos (OCDE) y de América Latina. El precio máximo regulado equivale al percentil 25 de menores precios de la muestra ${ }^{9}$.

La relación que se observa entre precios altos y la existencia de políticas de regulación de precios puede parecer contraria al sentido común. Mostraría que el control de precios no está logrando los efectos deseados en el conjunto de los medicamentos, o bien que las compañías farmacéuticas están reaccionando de un modo no previsto a los regímenes regulatorios. Una hipótesis alternativa, congruente con los resultados obtenidos, es que la regulación se hace más necesaria en economías que tienden a generar precios más altos.

Por otro lado, el uso de sistemas de precios de referencia (benchmarking) repercute en los precios de los países que sirven de referencia para regular los precios. En efecto, si un laboratorio desea bajar el precio de un medicamento en un determinado país que forma parte de la canasta de referencia de otro país, entonces el laboratorio deberá también reducir el precio de su medicamento en el país con precios regulados. Ello obviamente reduce los incentivos del laboratorio a bajar los precios, puesto que deberá traspasar al menos parte de dicha reducción a los países que cuentan con regulación. Un fenómeno análogo sucede con el alza de precios. El aumento de precios en países de referencia permite al laboratorio incrementar el precio del mismo medicamento en el país que cuenta con regulación. Esta sería otra razón para esperar que los precios de los países con regulación sean menores a aquellos que no fijan control alguno sobre los precios de los medicamentos.

Como se desprende de lo indicado, los efectos de la regulación de precios pueden ser variados y suscitar interesantes preguntas para la investigación. Con los datos de que se dispone, resulta difícil llegar a una conclusión respecto al impacto de la regulación en los precios, pero creemos que este trabajo aporta antecedentes de interés para investigaciones futuras sobre este tema.

\section{Conclusiones}

En el presente estudio se comparan los precios de medicamentos entre la Argentina, el Brasil, Chile. Colombia, México y el Perú. El ejercicio comparativo se realiza tanto para el total de medicamentos como para un nivel desagregado por las categorías comúnmente empleadas en el sector, a saber, productos innovadores, productos genéricos de marca o similares y productos genéricos puros.

\footnotetext{
8 Véase la Ley núm. 10.742, que "Define normas de regulação para o setor farmacêutico, cria a Câmara de Regulação do Mercado de Medicamentos - CMED" (Presidencia de la República, 2003). Los países de referencia son Australia, el Canadá, España, los Estados Unidos, Francia, Grecia, Italia, Nueva Zelandia, Portugal, el Reino Unido y el país de origen del medicamento.

9 Véase Comisión Nacional de Precios de Medicamentos y Dispositivos Médicos (2003).
} 
Se cuenta con un panel de observaciones realizadas mensualmente entre octubre de 2010 y noviembre de 2015, correspondientes a un total de 19.741 unidades vendidas en los seis países examinados, según datos proporcionados por IMS Health. Mediante el empleo de un modelo econométrico de panel con efecto fijo por país y el control de las variables referentes a las características y formato de venta de los productos, se obtuvo un ordenamiento de precios entre países. Las comparaciones se efectúan tanto en salida de fábrica como a nivel de venta al público final.

Nuestros resultados indican que a salida de fábrica y a nivel agregado de remedios, la clasificación de países del más barato al más caro es la siguiente: $1^{\circ}$ Argentina, $2^{\circ}$ Perú, $3^{\circ}$ México, $4^{\circ}$ Chile, $5^{\circ}$ Colombia y $6^{\circ}$ Brasil. A este respecto, la posición de Chile no difiere en términos estadísticos de la México. En la venta al público final, solo cambian los lugares iniciales de la clasificación, siendo el más barato el Perú, seguido de México y luego la Argentina, mientras que el resto de los países mantiene su posición. En cuanto a la magnitud del diferencial de precios, en salida de laboratorio la diferencia entre el país más caro y el más barato es de un 54\%, valor que alcanzaría a un 60\% en salida de farmacia.

Siempre en salida de fábrica y en productos innovadores, la Argentina, el Brasil y Chile, en ese orden, presentan los menores precios en la muestra. En genéricos de marca, la Argentina, México y el Brasil son los más baratos, mientras que en genéricos puros Chile y el Perú comparten el primer lugar en precios más bajos, al no existir diferencia estadísticamente significativa en su posición. La ubicación de los países en la clasificación no se altera al realizar la comparación a nivel de precio al público para las tres categorías de medicamentos analizadas.

Puede llamar la atención que los dos países que presentan un nivel de precios más alto a nivel agregado -el Brasil y Colombia- sean a su vez aquellos que mantienen sistemas formales de control de precios de medicamentos. Sin embargo, el análisis realizado no permite establecer una relación de causalidad entre ambos factores puesto que -como mencionamos-, las diferencias de precios entre países dependen de una serie de variables adicionales al régimen regulatorio, como por ejemplo los aranceles comerciales y el grado de competencia a nivel de productores y distribuidores finales. Por otro lado, la existencia de regulaciones puede surgir como respuesta a la existencia de precios altos, lo cual podría explicar la correlación entre ambos hechos.

\section{Bibliografía}

Balmaceda, C., M. A. Espinoza y J. Díaz (2015), "Impacto de una política de equivalencia terapéutica en el precio de medicamentos en Chile", Value in Health Regional Issues, vol. 8, Amsterdam, Elsevier.

Bradley, D. B. (2007), "International price comparisons for novel and follow-on drugs", Value in Health, vol. 10, $N^{\circ} 6$, Amsterdam, Elsevier.

Cameron, A. y otros (2009), "Medicine prices, availability, and affordability in 36 developing and middle-income countries: a secondary analysis", The Lancet, vol. 373, № 9659, Amsterdam, Elsevier.

Comisión Nacional de Precios de Medicamentos y Dispositivos Médicos (2003), "Circular número 03 de 2013", Bogotá, 21 de mayo [en línea] https://www.minsalud.gov.co/sites/rid/Lists/BibliotecaDigital/RIDE/ DE/DIJ/circular-03-de-2013.pdf.

Danzon, P. M. y M. F. Furukawa (2011), "Cross-national evidence on generic pharmaceuticals: pharmacy vs. physician-driven markets”, NBER Working Paper, № 17226, Cambridge, Oficina Nacional de Investigaciones Económicas (NBER).

Machado, M. y otros (2011), "International drug price comparisons: quality assessment", Revista Panamericana de Salud Pública, vol. 29, № 1, Washington, D.C., Organización Panamericana de la Salud (OPS).

OCDE (Organización de Cooperación y Desarrollo Económicos) (2011), "Pharmaceutical expenditure", Health at a Glance 2011: OECD Indicators, París, OECD Publishing.

Presidencia de la República (2003), "Lei № 10.742", Brasilia, 6 de octubre [en línea] http://www.planalto. gov.br/ccivil_03/Leis/2003/L10.742.htm.

Productivity Commission (2001), International Pharmaceutical Price Differences, Canberra, Commonwealth of Australia [en línea] https://www.pc.gov.au/inquiries/completed/pharmaceutical-prices/report/pbsprices.pdf. 


\section{Anexo A1}

\section{Test de Fisher}

A continuación se presentan los resultados del test de Fisher (Test F) de Chile con respecto al resto de los países, correspondiente a cada muestra. Para interpretar los valores, se debe tener en cuenta que los valores de probabilidad mayores a 0,05 no rechazan la hipótesis nula de que las medias son estadísticamente iguales, con un 95\% de confianza.

Por ejemplo, para el cuadro de precio público:

- Para la muestra total, el coeficiente de Chile, que presenta un 95\% de confianza, es estadísticamente diferente al coeficiente del Brasil y del Perú, pero no al coeficiente de México y Colombia.

Para el cuadro de precio en salida de fábrica:

- Para la muestra total, el coeficiente de Chile, que presenta un 95\% de confianza, es estadísticamente distinto al coeficiente del Brasil y de Colombia, pero no al coeficiente de México y el Perú.

El hecho de que las diferencias entre dos coeficientes no sean estadísticamente distintas significa que no se puede decir nada concluyente respecto a estas diferencias. En una clasificación podrían estar ubicadas en el mismo lugar ya que sus distribuciones no son tan diferentes.

Cuadro A1.1

Test de Fisher en precios al público

\begin{tabular}{lcccc}
\hline Prob $>\mathrm{F}$ & Precio al público & & & \\
\hline Chile & Muestra total & Genéricos & Innovaciones & Similares \\
\hline Brasil & 0,0111 & 0,0000 & 0,7455 & 0,1660 \\
\hline Colombia & 0,0546 & 0,0001 & 0,0000 & 0,0002 \\
\hline México & 0,4125 & 0,0000 & 0,0108 & 0,0156 \\
\hline Perú & 0,0373 & 0,0162 & 0,0500 & 0,5196 \\
\hline
\end{tabular}

Fuente: Elaboración propia, sobre la base de datos de IMS Health.

Cuadro A1.2

Test de Fisher en precios de salida de fábrica

\begin{tabular}{lcccc}
\hline Prob $>\mathrm{F}$ & En fábrica & & & \\
\hline Chile & Muestra total & Genéricos & Innovaciones & Similares \\
\hline Brasil & 0,0244 & 0,0000 & 0,0091 & 0,6673 \\
\hline Colombia & 0,0404 & 0,0000 & 0,0000 & 0,0001 \\
\hline México & 0,5528 & 0,0000 & 0,0104 & 0,0186 \\
\hline Perú & 0,2158 & 0,6341 & 0,3485 & 0,0303 \\
\hline
\end{tabular}

Fuente: Elaboración propia, sobre la base de datos de IMS Health. 